\title{
Construction of a Practical SCAR Marker Linked to Clubroot Resistance in Chinese Cabbage, with Intensive Analysis of HC352b Genes
}

\author{
Nobuaki Hayashida $^{1 *}$, Yuri Takabatake ${ }^{1}$, Norihiro Nakazawa ${ }^{1}$, Daisuke Aruga ${ }^{1}$, \\ Hiromitsu Nakanishi ${ }^{1}$, Goro Taguchi ${ }^{1}$, Koji Sakamoto ${ }^{2}$ and Etsuo Matsumoto ${ }^{3}$ \\ ${ }^{1}$ Division of Gene Research, Shinshu University, Ueda 386-8567, Japan \\ ${ }^{2}$ Takii Plant Breeding \& Experiment Station, Konan 520-3231, Japan \\ ${ }^{3}$ Nagano Vegetable \& Ornamental Crops Experiment Station, Nagano 381-1211, Japan
}

\begin{abstract}
A restriction fragment length polymorphism (RFLP) marker, $\mathrm{HC} 352 \mathrm{~b}$, has identified as closely linked to the $\mathrm{CRa}$ locus responsible for clubroot resistance $(C R)$ in a study dissecting CR loci in Chinese cabbage (Brassica rapa $L$. ssp pekinensis). Unfortunately, the RFLP pattern, including HC352b detected by the cDNA clone HC352, was complicated and confused in its interpretation because it represented multiple-copy loci. To provide a practical HC352b marker for CR breeding programs, a sequence characterized amplified region (SCAR) marker was constructed along the analysis of HC352 genes, followed by its evaluation for CR selection. Schematic characterization of signals was achieved by identification of $\mathrm{HC} 352$ homologous genes with their restriction sites, employing $C R a$-positive and -negative doubled haploid (DH) lines. Genomic sequence information from a set of HC352 homologous genes was analyzed to identify the $C R a$ linked paralog HC352b, followed by the successful designation of a SCAR marker. This correctly predicted the CR phenotypes of all tested individuals of $F_{2}$ and back-crossed progenies.
\end{abstract}

Key Words: clubroot resistance, HC352, paralog, sequence characterized amplified region (SCAR), $C R a$.

\section{Introduction}

Clubroot is one of the major diseases of Brassicaceae. It is caused by the parasitic infection of Plasmodiophora brassicae, a soilborne fungus, in the stem root of plants (Ludwig-Müller, 1999). Chinese cabbage (Brassica rapa L. ssp pekinensis) is highly susceptible to disease and frequently suffers destructive damage in infected fields. Several breeding programs have been successfully carried out to develop clubroot resistance (CR) cultivars of Chinese cabbage using European turnips (B. rapa ssp. rapifera) as sources of resistance (Hirai et al., 2004; Matsumoto et al., 1998). CR in turnips is considered to be qualitative and is grouped into several genotypical categories corresponding to the pathotype of $P$. brassicae (Matsumoto et al., 2005). It is important to monitor the inheritance of these resistant genes using molecular markers in the breeding of Chinese cabbage (Kuginuki et al., 1997; Matsumoto et al., 2005; Suwabe et al., 2003).

$C R a$ is the responsible locus for CR in a dominant

Received; March 23, 2007. Accepted; October 15, 2007.

* Corresponding author (E-mail: nobuaki@giptc.shinshu-u.ac.jp). manner. HC352b was reported to be the most useful DNA marker linked to $C R a$, together with $\mathrm{E} 49_{380}$ (Matsumoto et al., 2005). The random amplified polymorphic DNA (RAPD) marker E49 380 is located $4.6 \mathrm{cM}$ from $\mathrm{CRa}$ with coupling linkage. $\mathrm{HC} 352 \mathrm{~b}$ was mapped at $2.9 \mathrm{cM}$ from $\mathrm{CRa}$ on the opposite side of $\mathrm{E}_{4} 9_{380}$, and appeared to have repulsion linkage. HC352b was established as an RFLP marker with a probe derived from cDNA clone HC352 (Matsumoto et al., 1998). Although specific HC352b signals detected by cDNA show tight linkage to clubroot resistance, many unlinked signals were detected simultaneously. The appearance patterns of non-linked signals were complicated and confused, obstructing the practical use of $\mathrm{HC} 352 \mathrm{~b}$ in breeding programs. To construct a sequence characterized amplified region (SCAR) marker on a HC352b locus with the characteristics of usefulness, clarity and reproducibility, we analyzed the genomic information of the HC352 loci intensively in two doubled haploid (DH) lines, T136-8 (CRa) and K10 (a susceptible allele of $C R a$ ). 


\section{Materials and Methods}

\section{Plant materials}

As source material with $C R a$, a DH line, T136-8 (B. rapa L. ssp pekinensis), was used (Matsumoto et al., 2005). DH lines of B. rapa L. ssp pekinensis, K10 and Q5, were used as the parent as sources of a susceptible allele of $C R a$. K10 originated from the "CR Kanko" and does not have $C R a$. Q5 is a typical clubroot-susceptible line. The original resistance source of the parental line T136-8 is a member of the European Clubroot Differential (ECD) series, ECD 02 (B. campestris ssp. rapifera line AAbbCC).

Fourteen and 90 individuals of $F_{2}$ progeny between T136-8 $\times$ K10 and T136-8 $\times$ Q5 were used for DNA analysis and CR tests. $F_{1}$ between T136-8 and Q5 was crossed with Q5 to produce back-crossed (BC) populations. Each $\mathrm{F}_{2}$ and $\mathrm{BC}$ individual was selfed to produce the next generation for segregation analysis.

\section{DNA extraction}

DNA was extracted from the leaves of parental lines, $\mathrm{F}_{2}$ and $\mathrm{BC}_{1}$ plants, according to the cetyl trimethyl ammonium bromide (CTAB) method (Murray and Thompson, 1980).

\section{RFLP analysis}

RFLP analyses were performed as previously described using the cDNA clone HC352 (Matsumoto et al., 1998) and its subclones composed of EcoRI-digested fragments. Conditions of electrophoresis, membrane transfer, probe preparation, hybridization, washing and signal detection were described previously (Matsumoto et al., 1998).

\section{Cloning and sequencing}

Regular procedures were used for cloning and sequencing DNA fragments (Nakanishi et al., 2005). A cloning vector $\mathrm{pXcm}$, the vector for the TA cloning of PCR fragments, was used with a Ligation Kit (Nippon Gene, Tokyo, Japan). Sequencing was performed according to the manufacturer's instructions (ABI PRISM 310, Applied Biosystems, CA, USA). Analysis of sequence information was performed using the Genetyx software package (Software Development, Tokyo, Japan) and the BLAST search with DDBJ (http:// blast.ddbj.nig.ac.jp/top-j.html, October 11, 2007). The sequence data produced in this report were submitted to DDBJ

\section{PCR}

The standard protocol was used as follows: In a $10 \mu \mathrm{L}$ reaction solution were mixed $3 \mathrm{ng}$ of template DNA, $0.5 \mathrm{mM}$ of forward and reverse primer, respectively, $0.2 \mathrm{mM}$ dNTPs, $1 \mu \mathrm{L}$ of $10 \times$ reaction buffer, $1.0 \mathrm{mM}$ $\mathrm{MgCl}_{2}$, and 0.5 unit of Taq DNA polymerase (Promega Corporation, Madison, USA). Amplification was performed in a thermal cycler programmed as follows: $94^{\circ} \mathrm{C}$ for $4 \mathrm{~min}$, then 40 cycles at $94^{\circ} \mathrm{C}$ for $30 \mathrm{~s}$, at an annealing temperature for $1 \mathrm{~min}$ and at $72^{\circ} \mathrm{C}$ for $1 \mathrm{~min}$, followed by a final extension of $7 \mathrm{~min}$ at $72^{\circ} \mathrm{C}$. The annealing temperature for $\mathrm{F} 7-\mathrm{R} 11$ was $38^{\circ} \mathrm{C}$, and for F16-R13 it was $46^{\circ} \mathrm{C}$. Sequences of F7 and R11 were 5'-TGCTACACCAAAAGATTCGAGAT-3' and 5'GGATCTCTTCTGAATGTGATG-3'. F16 and R13 were designed as 5'-CTTTATAATGGCTACTATTTA3' and 5'-TGCTCATGAGTGTATAACTA-3'.

\section{CR tests}

Inoculation tests for resistance to clubroot were as previously reported (Matsumoto et al., 1998). As an inoculation source, we used the M85 isolate of $P$. brassicae, which was identified as race 2 according to Williams's classification (Williams, 1966). Selfed progeny of 14 and $90 \mathrm{~F}_{2}$ plants derived from $\mathrm{T} 136-8 \times$ $\mathrm{K} 10$ and $\mathrm{T} 136-8 \times \mathrm{Q} 5$, and $21 \mathrm{BC}_{1}$ plants produced from $\mathrm{F}_{1} \times \mathrm{Q} 5$ were used for $\mathrm{CR}$ tests. Twenty to 40 seeds of each $\mathrm{F}_{3}$ and the $\mathrm{BC}_{1} \mathrm{~S}_{1}$ population were sown in the pathogen-inoculated soil. Disease infection was rated 30 days after sowing. Evaluation of $\mathrm{CR}$ was based on the previous report (Matsumoto et al., 2005). $\mathrm{F}_{2}$ and $\mathrm{BC}_{1}$ individuals that segregated no susceptible plants in their selfed progeny were scored as homozygotes (A) for $C R a$, while those that segregated both resistant and susceptible plants were assessed as heterozygotes $(\mathrm{H}) . \mathrm{F}_{2}$ and $\mathrm{BC}_{1}$ individuals whose selfed progeny were thoroughly galled were scored as homozygotes (B) for susceptibility.

\section{Results}

\section{Genomic information from RFLP analysis}

Hybridization patterns of $\mathrm{HC} 352$ on the genomic DNAs of T136-8, K10, and their $F_{2}$ progeny were analyzed. T136-8 has $C R a$, only one locus with exclusive responsibility for $\mathrm{CR}$ in the cultivar. As shown in Figure 1A, HC352 detected several fragments that were both in the lanes of K10 and T136-8.

For detailed analysis, HC352 was divided into two subclones at the EcoRI site located in the cDNA (Fig. 1B), and hybridization patterns were also developed using these subclones (Fig. 2 and data not shown). Using subclone 2 as a probe, $4 \mathrm{~kb}$ and $5 \mathrm{~kb}$ signals are clearly segregated, suggesting physical maps of HC352 genes. A summary of signals detected in parental lines is interpreted as in the scheme shown in Figure 1B. It was necessary to assume that two or more loci were detected by $\mathrm{HC} 352$ in order to explain all of the hybridized signals. Here we judged one of these loci as HC352b that was found using other lines of Chinese cabbage (Matsumoto et al., 2005). The other locus should be HC352a.

Signals of $0.8 \mathrm{~kb}$ and $5 \mathrm{~kb}$ in length would reflect the existence of the HC352b-T allele. Unfortunately, however, if we use the full length of the HC352 cDNA clone, the signal at $0.8 \mathrm{~kb}$ is somewhat faint, and the 


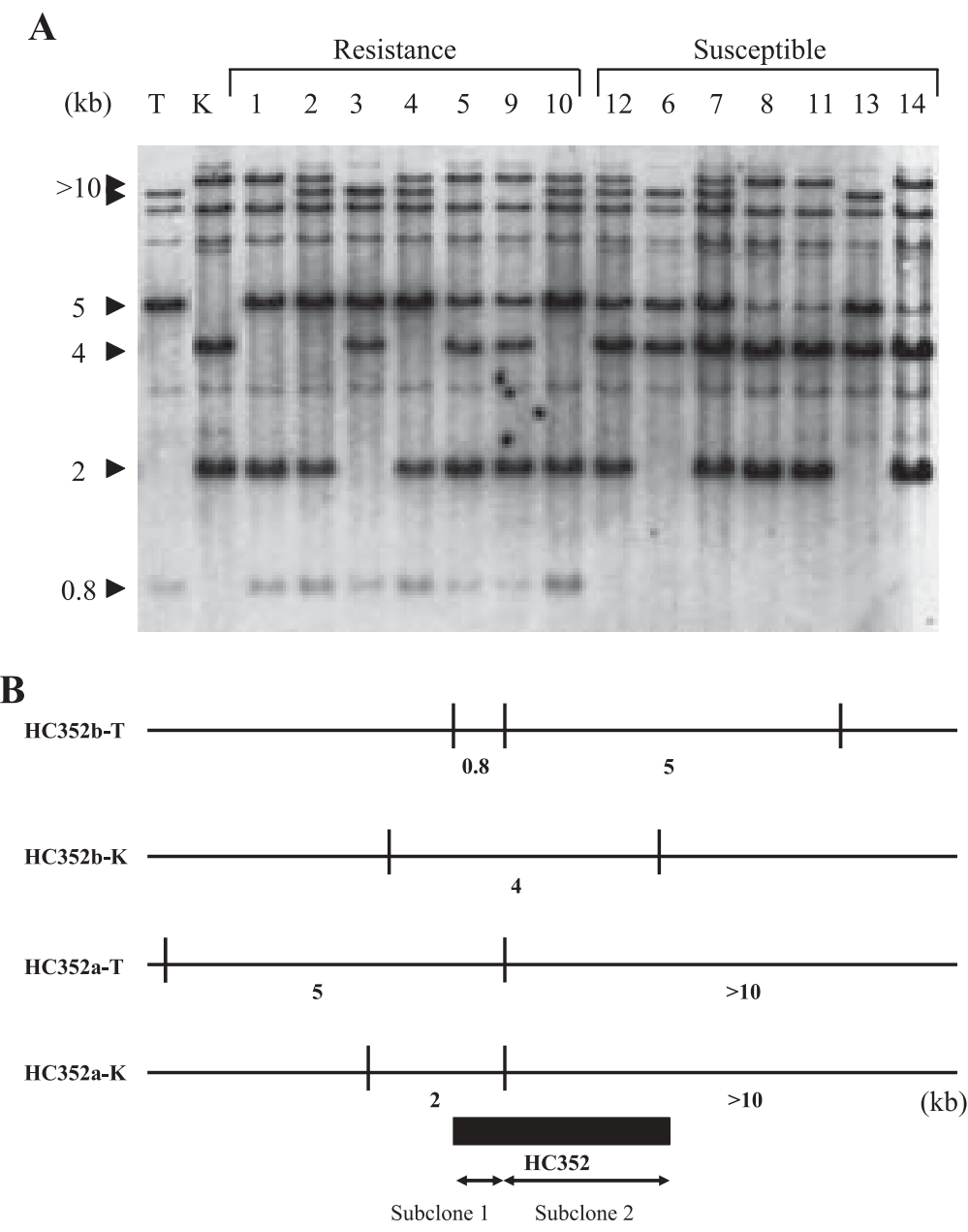

Fig. 1. Hybridization patterns of cDNA clone $\mathrm{HC} 352$ with the $\mathrm{F}_{2}$ population of $\mathrm{T} 136-8 \times \mathrm{K} 10$. A) The full length of the clone was used for the probe. Lanes $\mathrm{T}, \mathrm{K}, 1-5,9$, and 10 (resistance), and 6-8 and 11-14 (susceptible) indicate the genomic DNA of T136-8, K10, and the $\mathrm{F}_{2}$ individuals showing $\mathrm{CR}$ and non-CR, respectively. Each genomic DNA was digested by EcoRI. B) Scheme of the physical map of the HC352 genomic region. Horizontal lines indicate the genomic region containing the HC352 gene. The vertical line indicates EcoRI sites. Numbers correspond to the sizes of fragments detected in the hybridization. The filled box represents the position corresponding to the HC352 probe. Solid lines with arrowheads indicate positions of the subclones.

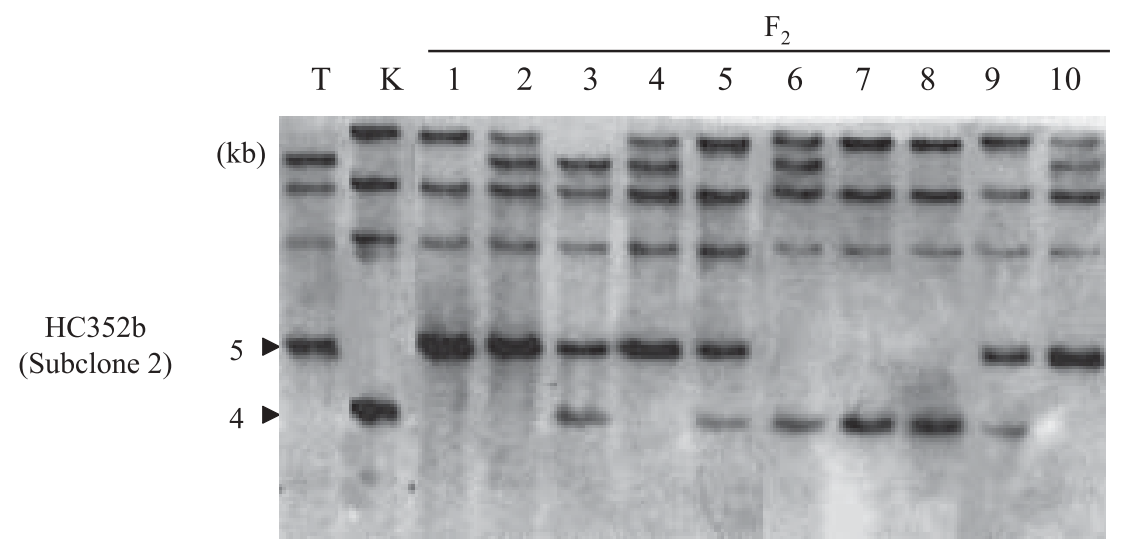

Fig. 2. Hybridization patterns of HC352 using subclone 2 of HC352 cDNA. RFLP was detected as in Fig. $1 \mathrm{~A}$ except that subclone 2 of HC352 cDNA was used as the probe. T: T136-8; K: K10; $F_{2}, 1-10$ : individuals of $F_{2}$ progeny of T136- $8 \times \mathrm{K} 10$.

signal at $5 \mathrm{~kb}$ is overlapped by another signal derived from HC352a-T.
Sequence information from cDNA clone HC352

The cDNA clone HC352 and two subclones were successfully sequenced and submitted to DDBJ 
(AB302983). Database searching analysis showed high homology with genes encoding RNase L inhibitor proteins. Two genes, At4g19210 and At3g13640, of Arabidopsis thaliana have the highest homology, and sequence AY185357 was recorded from Chinese cabbage. Since AY185357 was 3' partial EST, unfortunately it was not sufficient to be referred for the primer design. In the $A$. thaliana genome, two genes coding the RNase $\mathrm{L}$ inhibitor protein are located separately, i.e., in chromosomes IV and III. This coincides with our scheme of the HC352 regions.

\section{Sequence information from the HC352 regions of genomic DNA}

Primers F7 and R11 were designed on the positions of highly conserved sequences with two introns in the target sequence. This primer set brought a couple of fragments with PCR using genomic DNA (Fig. 3). The discrete fragments in Figure 3 were tentatively named

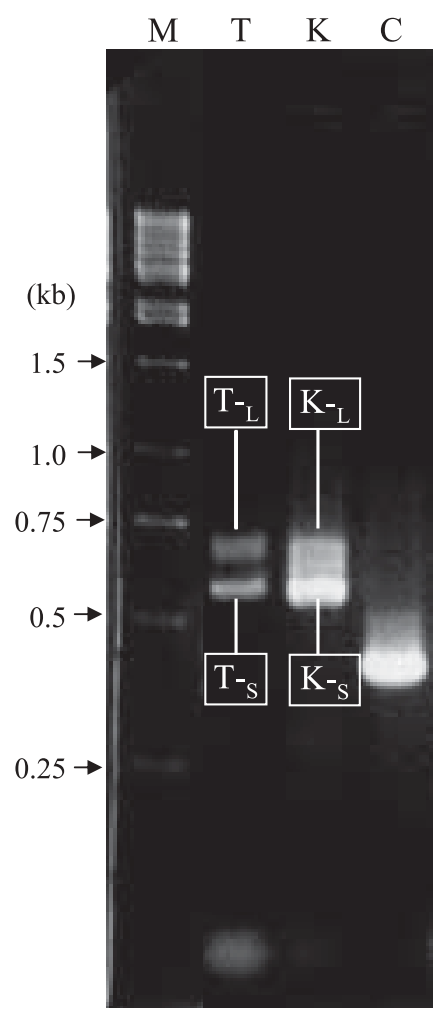

Fig. 3. PCR results using F7-R11 primers. Templates are T: genomic DNA of T136-8; K: genomic DNA of K10; C: HC352 cDNA clone. Fragments indicated as $\mathrm{T}_{-}, \mathrm{T}_{-}, \mathrm{K}_{-}$, and $\mathrm{K}_{-\mathrm{S}}$ are recovered from agarose gel for further analysis (see text). M: $1 \mathrm{~kb}$ DNA Ladder.
$\mathrm{T}_{-}, \mathrm{T}_{-\mathrm{S}}, \mathrm{K}_{-\mathrm{L}}$, and $\mathrm{K}-_{\mathrm{S}}$, and were sequenced after cloning $\left(\mathrm{T}_{\mathrm{L}}: \mathrm{AB} 302979 ; \mathrm{K}_{-}\right.$: AB302980; T-S: AB302981; K-s: AB302982). All of these fragments had good homology to part of the cDNA clone HC352, with two extra sequences considered to be introns. $\mathrm{T}_{-} \mathrm{L}$ has higher homology to $\mathrm{K}_{\mathrm{L}_{\mathrm{L}}}(98.8 \%)$ than to $\mathrm{T}-_{\mathrm{S}}(84.7 \%)$. Namely $\mathrm{T}_{{ }_{\mathrm{L}}}$ and $\mathrm{K}_{\mathrm{L}_{\mathrm{L}}}$ appear allelic, and $\mathrm{T}-_{\mathrm{S}}$ and $\mathrm{K}-\mathrm{s}$ could be allelic. T-s and $\mathrm{K}-\mathrm{s}$ were assumed to be $C R a$ linked (see Discussion), and were used for the further design of primers.

\section{Construction of the HC352b-SCAR marker}

HC352b-SCAR was designed to be amplified by $\mathrm{T}-\mathrm{S}$ specific primer set F16-R13, on the assumption that the diversity of $\mathrm{T}_{-\mathrm{S}}$ vs $\mathrm{K}_{-\mathrm{S}}$ is the substantial polymorphism of $\mathrm{HC} 352 \mathrm{~b}$. PCR with $\mathrm{F}_{2}$ from T136-8 $\times \mathrm{K} 10$ successfully amplified targeted fragments only from genomic DNAs of $\mathrm{F}_{2}$ individuals that have the $\mathrm{HC} 352 \mathrm{~b}$ signal in RFLP analysis (Figs. 2 and 4). Finally, linkage of the HC352bSCAR marker to the $C R a$ phenotype was demonstrated using the $\mathrm{F}_{2}$ population from $\mathrm{T} 136-8 \times \mathrm{Q} 5$ and the $\mathrm{BC}$ population from $\mathrm{F}_{1} \times \mathrm{Q} 5$ (Table 1). The HC352b-SCAR positiveness and the $\mathrm{CR}$ against M85 isolate ( $\mathrm{CRa}$ phenotype) were indicated to be tightly linked.

\section{Discussion}

In this report, we established the co-dominant SCAR marker HC352b-SCAR tightly linked to $C R a$, with intensive analysis of HC352-related genes. On the other hand, RAPD marker E49 380 was positioned at $4.6 \mathrm{cM}$ from $\mathrm{CRa}$ with coupling linkage (Matsumoto et al., 2005). HC352b-SCAR would be positioned at $2.9 \mathrm{cM}$ from $C R a$ and $7.5 \mathrm{cM}$ from $\mathrm{E} 49_{380}$, respectively, if it correctly detected "HC352b" as we expected. This result means that $C R a$ was closely put between dominant and co-dominant markers, providing efficient selection of $\mathrm{CR}$ plants in CR breeding programs.

Table 1. Linkage between HC352b-SCAR and $C R a$ phenotype.

\begin{tabular}{ccrrr}
\hline \hline \multirow{2}{*}{ Populations } & HC352b-SCAR & \multicolumn{3}{c}{ CRa phenotype } \\
\cline { 3 - 5 } & & $\mathrm{A}$ & $\mathrm{H}$ & $\mathrm{B}$ \\
\hline $\mathrm{F}_{2}$ & + & 23 & 60 & 0 \\
& - & 0 & 0 & 7 \\
\hline $\mathrm{BC}_{1}$ & + & - & 15 & 0 \\
& - & - & 0 & 6 \\
\hline
\end{tabular}

$\mathrm{F}_{1}$ and $\mathrm{F}_{2}$ plants were derived from $\mathrm{T} 136-8 \times \mathrm{Q} 5$. $\mathrm{BC}_{1}$ plants were produced from $\mathrm{F}_{1} \times \mathrm{Q} 5$. CRa phenotype was scored with $\mathrm{CR}$ tests using each $\mathrm{F}_{3}$ and $\mathrm{BC}_{1} \mathrm{~S}_{1}$ populations. A: CRa homozygous; $\mathrm{H}$ : $C R a$ heterozygous; B: Susceptible allele of $C R a$.

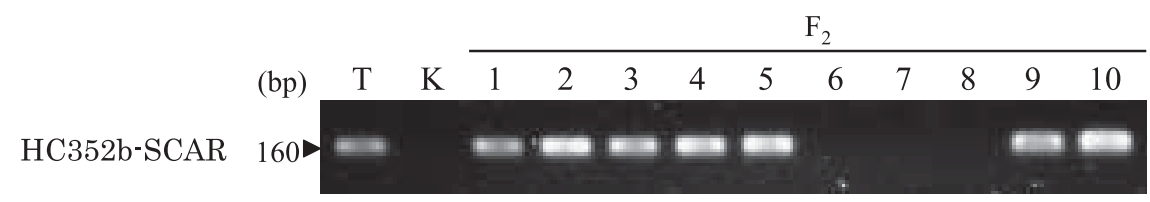

Fig. 4. Detection of HC352b-SCAR. HC352b-SCAR was detected with PCR using F16-R13 primer set. Material plants are the same as in Figure 2. 
The hybridization patterns of RFLP analysis coincided with reported data using segregated populations from crosses among several B. rapa cultivars and lines, including fodder turnips with a slight change in the size of fragments (Matsumoto et al., 2005). The $4 \mathrm{~kb}$ fragment in Figure 1 and 2 correspond to the $4.4 \mathrm{~kb}$ fragments detected in the susceptible lines as reported (Matsumoto et al., 1998, 2005). The scheme of restriction sites proposed in this report could explain those reported RFLP patterns, with the possibility that the scheme would also be applicable to other cultivars of Chinese cabbage and to turnips.

HC352 cDNA-specific primers were designed at several positions referring to sequences of the $A$. thaliana homologue. Amplified products using cDNA-specific primers are single fragments in every combination, with no polymorphism between T136-8 and K10 (data not shown), indicating that these primers could amplify only one of the loci detected by RFLP analysis. EcoRI digestion experiments with amplified fragments suggested that HC352a (Fig. 1B) had been amplified exclusively. The sequence of the cDNA clone HC352 coincides almost completely with that of $\mathrm{T}_{-}$and $\mathrm{K}_{-}$, indicating that the cDNA and $\mathrm{T}_{-} / \mathrm{K}_{-}$could be derived from allelic genes. Therefore, the cDNA clone HC352 and PCR fragments $\mathrm{T}_{-}$and $\mathrm{K}_{-}$were possibly derived from HC352a. We concluded that a simple effort to make direct conversion of the sequence information derived from the HC352 cDNA clone to the SCAR marker is not fruitful.

Analysis of the genomic sequence brought more insight into the constitution of HC352-related genes, supporting the scheme in Figure 1B. Here, the genomic region HC352b-T in Figure 1B may correspond to the $\mathrm{T}-\mathrm{s}$ fragment in Figure 3, and $\mathrm{HC} 352 \mathrm{~b}-\mathrm{K}$ may correspond to $\mathrm{K}_{-\mathrm{S}}$, HC352a-T to $\mathrm{T}_{-\mathrm{L}}$ and $\mathrm{HC} 352 \mathrm{a}-\mathrm{K}$ to $\mathrm{K}_{\mathrm{L}} \cdot \mathrm{K}_{\mathrm{L}}$ and $\mathrm{T}-_{\mathrm{L}}$ appear to be derived from allelic genes. $\mathrm{K}-\mathrm{S}$ is also be a counterpart of T- ${ }_{\mathrm{S}}$. In contrast, "HC352a" and "HC $352 \mathrm{~b}$ " genes are diverse genes that are related to each other as paralogs, having originated from the duplication of an ancestral gene. To confirm this possibility, the full sequences of HC352 genes are required.

Recently, large-scale duplications or multiplications in the genome of $B$. rapa were indicated in linkage map analysis (Kim et al., 2006). Synteny analysis with A. thaliana also provides evidence of multiplication in the Chinese cabbage genome (Saito et al., 2006). This information makes us cautious regarding the crossdetection of paralogical genes in studies of markerassisted breeding. To overcome this problem, resequencing of homologous genes might be a powerful tool, as in this work. The employment of divergence and polymorphism uncovered by intensive sequencing could become a stable and secure strategy.

\section{Acknowledgements}

We would like to thank Dr. Hatsumi Nozue for her valuable discussions.

\section{Literature Cited}

Hirai, M., T. Harada, N. Kubo, M. Tsukada, K. Suwabe and S. Matsumoto. 2004. A novel locus for clubroot resistance in Brassica rapa and its linkage markers. Theor. Appl. Genet. 108: 639-643.

Kim, J. S., T. Y. Chung, G. J. King, M. Jin, T. J. Yang, Y. M. Jin, H. I. Kim and B. S. Park. 2006. A sequence-tagged linkage map of Brassica rapa. Genetics 174: 29-39.

Kuginuki, Y., H. Ajisaka, M. Yui, H. Yoshikawa, K. I. Hida and M. Hirai. 1997. RAPD markers linked to a clubroot-resistance locus in Brassica rapa L. Euphytica 98: 149-154.

Ludwig-Müller, J. 1999. Plasmodiophora brassicae, the causal agent of clubroot disease: A review on molecular and biochemical events in pathogenesis. J. Plant Dis. Prot. 106: 109-127.

Matsumoto, E., N. Hayashida, K. Sakamoto and M. Ohi. 2005. Behavior of DNA markers linked to a clubroot resistance gene in segregating populations of Chinese cabbage (Brassica rapa ssp. pekinensis). J. Japan. Soc. Hort. Sci. 74: 367-373.

Matsumoto, E., C. Yasui, M. Ohi and M. Tsukada. 1998. Linkage analysis of RFLP markers for clubroot resistance and pigmentation in Chinese cabbage (Brassica rapa ssp. pekinensis). Euphytica 104: 79-86.

Murray, M. G. and W. F. Thompson. 1980. Rapid isolation of high molecular weight plant DNA. Nucl. Acid. Res. 8: 43214325.

Nakanishi, H., H. Nozue, K. Suzuki, Y. Kaneko, G. Taguchi and N. Hayashida. 2005. Characterization of the Arabidopsis thaliana mutant pcb2 which accumulates divinyl chlorophylls. Plant Cell Physiol. 46: 467-473.

Saito, M., N. Kubo, S. Matsumoto, K. Suwabe, M. Tsukada and M. Hirai. 2006. Fine mapping of the clubroot resistance gene, Crr3, in Brassica rapa. Theor. Appl. Genet. 114: 81-91.

Suwabe, K., H. Tsukazaki, H. Iketani, K. Hatakeyama, M. Fujimura, T. Nunome, H. Fukuoka, S. Matsumoto and M. Hirai. 2003. Identification of two loci for resistance to clubroot (Plasmodiophora brassicae Woronin) in Brassica rapa L. Theor. Appl. Genet. 107: 997-1002.

Williams, P. H. 1966. A system for the determination of races of Plasmodiophora brassicae that infect cabbage and rutabaga. Phytopathol. 56: 624-626. 